\title{
MinORÍAS ÉTNICO-CULTURALES Y DERECHOS DE LAS MUJERES ${ }^{*}$
}

Ethno-Cultural Minorities and Women's Rights

\section{LUIS VILLAVICENCIO MIRANDA}

\author{
Universidad de Valparaíso
}

\begin{abstract}
RESUMEN
Este artículo examina las bases de una propuesta conciliatoria que permita equilibrar las pretensiones del feminismo de la igualdad con una noción de la ciudadanía suficientemente inclusiva, deteniéndose en particular en la propuesta de las jurisdicciones multiculturales. Para ello, en primer lugar, se revisa el modo en que el feminismo de la igualdad y el multiculturalismo coinciden en una crítica a la noción tradicional de ciudadanía liberal. En segundo lugar se indaga la posibilidad de construir una concepción que permita superar ese ideal de ciudadanía. Finalmente se analiza críticamente la tesis de las jurisdicciones multiculturales para concluir que por sí sola no es suficiente, debiendo insertarse en una adecuada concepción de la democracia deliberativa.
\end{abstract}

Palabras clave: Feminismo, multiculturalismo, ciudadanía, jurisdicciones multiculturales, democracia deliberativa.

\begin{abstract}
This article examines the foundation of a conciliatory proposal that balances the claims of equality feminism with a notion of an inclusive citizenship that consider the multicultural jurisdictions approach. We first review how equality feminism and multiculturalism agree on a critique of a traditional liberal citizenship notion. Second, we explore the possibility of building a conception that allows overcoming that ideal of citizenship. Finally, it analyses critically the thesis of the multicultural jurisdictions for conclude that it alone is not enough and it must be inserted into an adequate conception of deliberative democracy.
\end{abstract}

Key words: Feminism, multiculturalism, citizenship, multicultural jurisdictions, deliberative democracy.

* El presente trabajo forma parte del Proyecto FONDECYT No 1120566 titulado “Ciudadanía para las mujeres en una sociedad multicultural. Hacia la construcción de una concepción deliberativa con vocación universal y su impacto institucional". El autor agradece los comentarios de Rodolfo Figueroa, coinvestigador del proyecto; de la profesora Alejandra Zúñiga Fajuri; la colaboración de los ayudantes del proyecto Felipe Pérez y Cecilia Valenzuela, licenciados en Ciencias Jurídicas y Sociales de la Universidad de Valparaíso; y de los tesistas Francisca Marchant Letelier y Cristián Martínez Vera, también de la Universidad de Valparaíso. 


\section{INTRODUCCIÓN}

Un aporte relevante del feminismo de la igualdad ${ }^{1}$ ha sido la revisión de las categorías conceptuales empleadas en la filosofía política, moral y jurídica. "Desde este punto de vista, la tarea emprendida por el feminismo [ilustrado] ha supuesto tanto una deconstrucción de conceptos que se postulaban normativa y descriptivamente como universales -tal y como sería el caso del sujeto político o el sujeto de derechos- desvelando así el subtexto de género implícito en ellos, como por otro lado, una reconstrucción de los marcos categoriales ya establecidos y la elaboración de propuestas teóricas alternativas" (Sánchez, 2005: 586). ${ }^{2}$

En el ámbito de la filosofía política en particular, la revisión de la ciudadanía es una de las preocupaciones que más ha captado la atención de la investigación feminista ilustrada (Cfr. Deveaux, 2006: 54-88). Si bien el feminismo de la igualdad puede definirse como "un tipo de pensamiento antropológico, moral y político que tiene como su referente la idea racionalista e ilustrada de la igualdad entre los sexos" (Amorós, 1997: 70) -y, por lo tanto, su origen se remonta a los albores mismos de la Ilustración para denunciar el universalismo de carácter sustitucionista que desconoce al "otro concreto" (Benhabib, 2006b: 189)- una vez que las mujeres pasaron a formar parte del demos de la comunidad política, la teoría feminista se ha fragmentado en múltiples tendencias que abordan, desde diferentes ángulos, la vieja demanda de hacer patentes las persistentes desigualdades en las relaciones de poder entre hombres y mujeres por el control, los primeros, de la fuerza de trabajo, de las segundas.

En ese escenario se ha producido un desplazamiento en la teoría feminista desde posiciones universalistas hacia aquellas identificadas con la afirmación de las diferencias (Cfr. Sánchez, 2002: 347-370). Ese alejamiento se ha desatado en un entorno en el que las políticas de la diferencia, que se alientan desde las huestes multiculturalistas, también se han agudizado ${ }^{3}$, al punto que, para algunos autores, las políticas de redistribución que caracterizan al estado democrático de derecho se encuentran notablemente amenazadas. ${ }^{4}$

1 Este no es un artículo que pretenda analizar exhaustivamente la teoría feminista. Ello no es posible ni por su extensión, ni por su objetivo. Con afán nada más ilustrativo puede revisarse Hesse-Biber (2012) para observar la diversidad y multiplicidad de aproximaciones feministas a los desafíos teóricos y prácticos que nos demandan las sociedades modernas.

2 Sobre la relación entre género y ciudadanía véase, en nuestro medio, Zúñiga $(2009,2010)$.

3 Obviamente el hecho de la diversidad cultural no es una novedad. Sin embargo, tres circunstancias hacen particularmente apremiante la articulación de renovadas propuestas que se hagan cargo de este desafío: a) la expansión de la concepción democrática del Estado ha permitido a las comunidades minoritarias reclamar igual derecho a participar en el autogobierno colectivo; b) el proceso de globalización económica y cultural ha pulverizado cualquier intento de asimilación a gran escala; y c) la ilusión del Estado culturalmente homogéneo ha terminado por sucumbir. Cfr. Parekh (1999); Kymlicka (2002: 327-328) y Torbisco (2006: 1-9). Frente al desafío multicultural se han erigido tres grandes respuestas teóricas: la del liberalismo igualitario, la del multiculturalismo liberal, y las diferentes formas de multiculturalismo. Cfr. Pérez de la Fuente (2005:254). Para un análisis general puede verse Villavicencio (2010a). Para un examen del liberalismo como una perspectiva que fracasa en su intento véase Villavicencio (2009 y 2010b).

4 Esa es, por ejemplo, la posición de Barry. El filósofo inglés considera positivo que el liberalismo sea "ciego a la diferencia", ya que los liberales no buscan erradicar las formas de vida tradicionales ni reclamar contra 
La cuestión central que se propone este trabajo es explorar las bases de una propuesta conciliatoria que permita equilibrar las pretensiones de una ciudadanía más inclusiva que propugna el feminismo de la igualdad deteniéndome, en particular, en la propuesta de las jurisdicciones multiculturales de Shachar (2001). Como lo plantea la autora, el dilema básico radica en cómo dividir, en un Estado multicultural, la autoridad de manera equitativa logrando un equilibrio entre la acomodación de las tradiciones de grupos minoritarios y la protección de los derechos individuales de ciudadanía. La tesis fundamental es la construcción de una concepción de la ciudadanía diferenciada guiada por un ambicioso principio innovador: "uno que se esfuerza por la reducción de la injusticia entre grupos, junto con la mejora de la justicia dentro de ellos" (Shachar, 2001: 4). Desde luego, la posibilidad a la que se alude es, al menos en principio, remota. Pero que lo improbable devenga en probable es tarea habitual de la filosofía.

\section{DOS CASOS QUE ILUSTRAN LA TENSIÓN ENTRE LA CULTURA Y LOS DERECHOS DE LAS MUJERES}

El 2011 la Corte de Apelaciones de Santiago de Chile desestimó una acción de protección presentada por una mujer musulmana, en contra de una sucursal del Banco Estado, por habérsele exigido levantarse el hiyab, bajo el pretexto de que no había otro modo de verificar su identidad, y así poder cobrar un cheque. La recurrente alegó que se le había dado un trato vejatorio, humillante y discriminatorio, vulnerando su libertad religiosa. Si bien la mayoría rechazó el amparo constitucional, el voto disidente argumentó que el velo era más que un símbolo estrictamente religioso para transformarse en un "principio de identidad cultural [pues la] (...) mujer que en una realidad como la chilena se desplaza públicamente con hiyab, explicita con orgullo su pertenencia a lo que juzga más propio, a saber, la civilización islámica". ${ }^{5}$ Lo destacable del razonamiento apuntado es que concibe al velo islámico como algo más que una prenda religiosa y, por lo tanto, resalta la circunstancia de que resolver el caso nada más concentrándose en la colisión entre dos o más derechos fundamentales implica adoptar una perspectiva reduccionista que las posiciones multiculturalistas se han cansado de denunciar.

En Francia, paradigma del republicanismo asimilacionista y laicista, entró en vigencia el 11 de abril de 2011 una normativa que prohíbe el uso del velo integral islámico (burka y niqab) en espacios públicos. El argumento central para justificar la imposición es que este tipo de vestimenta constituye uno de los símbolos más relevantes de la opresión de la mujer en nombre de la cultura tradicional y del Islam fundamentalista. Sus detractores

la mantención de las culturas ancestrales, sino simplemente oponerse a la coerción respecto de los que no comparten estos objetivos (2002: 66). No están a favor ni en contra de la asimilación, sino simplemente creen que la cantidad justa de ella es la que se da en un marco de instituciones equitativas. Hemos defendido, en otra parte, que las políticas de la diferencia no son incompatibles con las políticas de redistribución, véase Selamé y Villavicencio (2011).

5 Fundamento segundo del voto en contra del ministro Cerda. El fallo puede consultarse en: http:// diarioconstitucional.cl/articulo-det.php?id_articulo=1409\&id_cat=9. 
alegan -entre ellos muchas de las propias mujeres que utilizan esas prendas- que se está vulnerando la libertad religiosa y presumiendo que, en ningún caso, constituye una decisión libre utilizarlas. ${ }^{6}$

Mediante estos ejemplos busco resaltar algunas cuestiones relevantes para el tema que aquí se trata: a) en primer lugar, el nexo que tienen las personas -tanto como agentes morales individuales y como grupo- con la cultura ${ }^{7}$; b) en segundo lugar, el hecho de que esa vinculación es problemática y compleja, pero necesaria; y c) en tercer lugar, dicha vinculación necesaria debería ocuparse de construir una vía alternativa al universalismo sustitutivo y excluyente (Benhabib, 2006b: 171-201), ${ }^{8}$ pero también al relativismo cultural tributario de una perspectiva multicultural esencialista que es utilizado, además, en muchas partes del mundo como excusa para todo tipo de violaciones de los derechos de las mujeres.

Termino este apartado refiriéndome, brevemente, a la idea de "cultura" que subyace a este trabajo. Cuatro son los aspectos que me interesa destacar:

a) La idea del proceso. Apoyándome en Phillips (2007b: 15-29), la cultura podemos comprenderla como lo "cotidiano" u "ordinario". Aquel proceso omnipresente, continuo y cambiante en donde cualquier organización social se desarrolla y se reproduce a sí misma construyendo un significado compartido, transmitido de generación en generación y abierto al debate y enmiendas.

b) El choque cultural como una distribución desigual del poder. Desde el punto de vista de la filosofía política, la cultura importa no tanto como el estudio de la diferencia de las prácticas culturales, sino como desigualdad, es decir, determinar qué cuenta como justo tratamiento para los grupos minoritarios. Luego, el reconocimiento de las relaciones de poder desiguales entre los grupos minoritarios y mayoritarios junto con la percepción de que los Estados casi siempre discriminan a los ciudadanos de grupos culturales minoritarios, al imponer una política unitaria y un marco legal, es lo que aquí me interesa destacar. Indicadas esas características, no debe sorprendernos que los grupos minoritarios estén, a menudo, en conflicto con el grupo dominante.

6 Un proceso similar se está produciendo en España. “Lleida fue el primer municipio español en proponer la prohibición del burka y ha sido también el primero en saber que la justicia acepta su decisión". Noticia publicada en El País de 10 de junio de 2011.

7 Este punto podrá parecer obvio, pero no debemos olvidar que hasta hace no mucho, bajo el monopolio de la concepción liberal de ciudadanía, el agente moral era definido como anterior a sus fines y, por lo mismo, desvinculado de los diferentes marcos cualitativos culturales que definen su identidad. Para una revisión crítica de esta tesis liberal en retirada véase Villavicencio (2007). Probablemente el comunitarismo y el feminismo sean las dos corrientes que más claramente le han reprochado al liberalismo su excesiva abstracción. Walzer escribe, desde las filas comunitaristas, que "[la pregunta no es] ¿qué escogerían individuos racionales en condiciones universalizantes de tal y tal tipo?, sino ¿qué escogerían personas como nosotros, ubicadas como nosotros lo estamos, compartiendo una cultura y decididos a seguirla compartiendo?" (1993: 19). Respecto del feminismo puede verse Frazer y Lacey (1993: 53-77).

8 Ejemplos paradigmáticos de ese tipo de universalismo serían los casos del primer Rawls (el de $A$ Theory of Justice) y de Habermas. Sobre este punto véase Sánchez (2007 y 2008). 
c) El proceso de significación radica en los agentes, no en la cultura. Por su parte Dhamoon (2007: 30-49), basándose en la semiótica, define lo cultural como un proceso de construcción de significado. Este énfasis es decisivo, pues modifica el análisis desde el objeto de la cultura distinta (la entidad que tiene un significado) al proceso que expresa esa identidad diversa. El cambio supone comprender que aunque los signos no son permanentes o fijos, no es la cultura en sí misma la que está en riesgo, sino las autodeterminaciones y posiciones de los agentes (como individuos o grupos) para hacer sus propios sistemas de construcción de significado y moldear sus propios significados en contextos de no subordinación.

d) Las culturas tienen potestades normativas. El último elemento de la noción de cultura es lo que Shachar (2001: 2) denomina como comunidad nomoi. Con ello se refiere a un grupo que tiene una visión del mundo comprensiva y distinguible que se concreta en potestades normativas que crean leyes para la comunidad. Estos grupos se distinguen no solo por sus particulares sistemas de significado, sino por pretender regular por medio de la ley la conducta de la comunidad de sus miembros.

\section{EL FENÓMENO DEL DESPLAZAMIENTO: LA ATOMIZACIÓN DE LA REIVINDICACIÓN DE LA CIUDADANÍA}

Tanto para la teoría feminista ${ }^{9}$ como para las diversas clases de multiculturalismos, ${ }^{10}$ la concepción liberal de la ciudadanía fomenta la marginación o estigmatización de grupos que escapan del estereotipo en el que se funda la ficción del Estado nacional. Esa noción de ciudadanía tradicional se caracteriza por considerar a los individuos como la fuente última de la legitimidad política, dándoles la palabra final sobre la determinación del sistema político y el contenido de sus políticas, pero al mismo tiempo -y en evidente tensión con lo anterior- concibiéndolos como titulares de ciertos derechos y libertades inalienables por su condición de agentes morales abstractamente iguales. ${ }^{11}$

Los derechos ideados para ese "ciudadano normal" no se acomodan a las necesidades de grupos diferenciados, pues demandan algo más que las tradicionales políticas distributivas del Estado democrático de derecho. Esto se explica porque al interior de

9 No se me escapa que, a pesar de que existen muchas formas de feminismo, la teoría feminista moderna converge abstractamente en una misma gran demanda: la revisión crítica de la idea de ciudadanía y su universalidad excluyente. Cfr. Sánchez (2002).

10 No hay discusión respecto de que Kymlicka es el pensador más representativo de lo que él mismo llama multiculturalismo liberal (Kymlicka, 2007: 61-86). Sin embargo, tratándose del multiculturalismo a secas, la cuestión es más complicada, ya que carece de fundadores o textos canónicos (Parekh, 2002: 133-150). Por esta razón, parece más adecuado hablar de multiculturalismos (De Lucas, 2001: 61-102; Banting y Kymlicka, 2006: 9), que solo tendrían en común la defensa de una estrategia basada en el paradigma del reconocimiento (Young, 2000: 31-69).

11 Song (2007: 68). Sobre la idea de ciudadanía en general vinculado con el problema de la diversidad cultural y el género puede verse Delanty (2010), Jackson (2005), y Modood (2007). En nuestro medio es imprescindible el análisis de Zúñiga (2010). 
los Estados modernos conviven dos tipos principales de jerarquías: la económica y la asociada al estatus. La posición que una persona ocupa en la jerarquía económica está determinada por su relación con el mercado y los medios de producción. La lucha contra las iniquidades inherentes a esta jerarquía genera las políticas de redistribución. La jerarquía del estatus se refleja en una historia de reglas discriminatorias contra grupos de menor categoría, y su invisibilidad o carácter estereotipado. La lucha contra estas jerarquías genera las políticas de reconocimiento o de la diferencia (Cfr. Fraser, 2000; Delanty, 2010: 59; y Modood, 2007: 68-70).

A pesar de que podamos distinguir las políticas de redistribución y reconocimiento para fines analíticos, lo cierto es que en el mundo real aparecen a menudo superpuestas (v. gr. piénsese en las mujeres mapuche que son al mismo tiempo una minoría sexual, nacional, étnica y social). Con todo, la evidencia sugiere que la jerarquía del estatus no es reducible a la jerarquía económica. Como prueba de lo anterior, podemos señalar casos de grupos económicamente bien posicionados, pero culturalmente estigmatizados, como los homosexuales, ciertos inmigrantes y algunos grupos religiosos; $\mathrm{y}$, a la inversa, casos de grupos que gozan de una posición privilegiada en la jerarquía del estatus, pero que se encontraban hasta hace poco en desventaja económica, como la clase trabajadora masculina en la mayoría de las democracias occidentales. No hay, entonces, una correlación simple entre ambas jerarquías. Esto explica por qué la estrategia de una ciudadanía común funcionó para la clase trabajadora masculina, pero no satisfizo a otros grupos, las mujeres sin ir más lejos, quienes necesitaban, además, un ataque a las jerarquías sustentadas en el estatus (Kymlicka, 2002: 327-376).

En esa línea, el planteamiento de Young gira en torno a un nuevo concepto de opresión que "designa las desventajas e injusticias que sufre alguna gente no porque un poder tiránico la coaccione, sino por las prácticas cotidianas y estructurales de una bien intencionada sociedad liberal". En particular, tratándose de las mujeres, la opresión consiste, en parte, en una transferencia sistemática y no recíproca de poderes a los hombres, que implica más que una desigualdad de estatus, poder y riqueza resultante de la práctica cultural por la que los hombres han excluido a las mujeres de las actividades privilegiadas, ya que esa desigualdad es posible precisamente porque las mujeres trabajan para ellos. De este modo, la explotación de género se inicia con la transferencia a los hombres de los frutos del trabajo material y de las energías sexuales y de crianza de las mujeres, lo que no se modificará a menos que lo haga la estructura social y cultural (Young, 2000: 89-93).

Ahora bien, si con algo se ha enfrentado la teoría y los movimientos feministas ilustrados es, precisamente, con la cultura. Algunas autoras asumen una postura dicotómica, pues denuncian que la cultura opera en demasiados casos como una "pseudojustificación" para violar los derechos de las mujeres (por ejemplo, la ablación genital, el planchado de pechos, la lapidación, la violación como mecanismo reparatorio, el tráfico sexual, los matrimonios concertados, el rapto de novias, entre los más relevantes). Si al analizar las conexiones entre cultura y género nos cuestionamos sobre el efecto que juegan las restricciones culturales sobre la vida de hombres y mujeres para percatarnos que los primeros son, casi siempre, los beneficiados y las segundas abrumadoramente perjudicadas, 
sencillamente no podemos aceptarlas (Cfr. Guerra, 2000: 101-116). Así parece ineludible adoptar el punto de vista de que no hay otra opción que una trágica y simple elección entre tu cultura o tus derechos (Shachar, 2001: 2 y 3; y Okin, 1999). ${ }^{12}$ Esa sería, sin ir más lejos, la justificación de la prohibición francesa del velo integral.

Por otro lado, varios autores plantean que las reivindicaciones culturales no son incompatibles, en general, con las demandas de autonomía política y moral (Parekh, 2002: 133-150; y 2006: 165-172) ni, en particular, con los requerimientos de libertad e igualdad de las mujeres (Benhabib, 2002: 172-175). Específicamente sobre estas últimas reclamaciones la filósofa turca reflexiona:

la autonomía y el pluralismo cultural parecen irreconciliables. ¿Pero esto debe ser así necesariamente? No hay duda de que las preocupaciones de las mujeres y el estatus de la esfera privada exponen la vulnerabilidad de los acuerdos multiculturales y ponen de manifiesto los injustos compromisos políticos y morales, logrados a expensas de mujeres, niños y niñas, sobre los cuales a menudo descansan ¿Pero qué sucedería si ambas posiciones se basaran en presupuestos epistemológicos equivocados respecto de las culturas (...)? Si adoptáramos la perspectiva del diálogo multicultural complejo por la que abogo, ¿podríamos ser capaces de hacer justicia tanto a las aspiraciones de libertad e igualdad de las mujeres como a la legítima pluralidad de las culturas humanas? (Benhabib, 2002: 101).

Para esta postura el antagonismo sin más entre la igualdad de género y la perspectiva multicultural es caricaturesco y excesivo, pues la cultura sí importa y deben buscarse mecanismos para vindicar la ciudadanía de las mujeres en un mundo irremediablemente multicultural. Con matices, esa es la postura que subyace al voto disidente del fallo de la Corte de Apelaciones de Santiago citado más arriba.

\section{BUSCANDO UNA CONCEPCIÓN INTEGRADORA}

Siguiendo a Fraser (2000), podemos identificar dos serias dificultades a propósito de las demandas de reconocimiento que deben ser neutralizados sin desmerecer la justicia de algunas de esas reivindicaciones: el problema del desplazamiento y el problema de la cosificación. Según el primero, los planteamientos a favor del reconocimiento están desplazando las luchas a favor de la redistribución. De acuerdo con el segundo, se produce una sobrecosificación de la identidad cultural o grupal que acarrea el peligro de justificar la violación de derechos básicos en nombre de categorías identitarias petrificadas que congelan los antagonismos que pretenden remediar. En esas circunstancias no debe sorprendernos que muchas personas sencillamente se hayan olvidado de las luchas culturales (v. gr. Barry, 2002). Estas últimas reacciones son muy comprensibles, pero al mismo tiempo gravemente erróneas: por una parte, no todas las formas de políticas de la diferencia son igualmente perniciosas, pues muchas de ellas representan respuestas

12 Para ser exacto, Okin relativizó su postura en trabajos posteriores reconociendo que el título provocativo de su ensayo llevó al error a sus lectores críticos. Véase Okin (2005). 
genuinamente emancipatorias frente a graves injusticias estructurales; $y$, por otra, la cultura constituye un terreno de lucha legítimo que visibiliza aquellas formas de injusticia que no podemos ver bajo las categorías tradicionales, apareciendo en muchas ocasiones conectadas implícitamente con desigualdades económicas. En otras palabras, defender la conexión entre la idea de identidad y las políticas de la diferencia o reconocimiento no implica confundirlas con las políticas de la identidad esencialistas (Squires, 2002: 114-132).

Si lo que vengo sosteniendo es cierto, esa conexión entre las mujeres y la cultura nos sitúa en la complicada posición de redimir una ciudadanía inclusiva para las mujeres, en un mundo cada vez más multicultural, que pueda hacerse cargo de la siguiente interrogante: ¿cómo compatibilizar las acomodaciones multiculturales con las pretensiones universalistas de los derechos individuales liberales? Para responder esa pregunta me parece fundamental dar una vuelta de tuerca a los enfoques feministas ilustrados más relevantes, ya que se encontrarían en pie forzado: sus reivindicaciones no encuentran una respuesta adecuada en el modelo de ciudadanía no diferenciada que propugna el liberalismo igualitario; pero, al mismo tiempo, si bien los presupuestos multiculturales les permitirían sustentarse en un marco conceptual que admitiría considerar adecuadamente las diferencias de género, necesitan imperiosamente buscar mecanismos para blindarse ante las brutales vulneraciones de los derechos más básicos de las mujeres que se cometen en nombre de la identidad cultural. ${ }^{13}$

En pocas palabras, al feminismo ilustrado no le queda otro camino que conciliar algunas (no todas) de las tesis multiculturalistas con ciertas (no todas) tesis liberales que deben ir más allá del simple derecho de salida (abandono de la propia cultura) que genera un costo demasiado severo para aquel o aquella que decide alejarse (Phillips: 2007a: 151-157).

Analíticamente podemos agrupar tres clases de respuestas teóricas a la pregunta que he planteado:

a) La respuesta liberal igualitaria que busca re-universalizar la ciudadanía, de manera de incluir genuinamente a las mujeres profundizando el paradigma de la redistribución de género. Ese sería el caso de Barry o Habermas (1999: 189-227; y 2003: 4-10) y, dentro del feminismo liberal, por ejemplo, la apuesta de Okin y Nussbaum (1999: 105-114);

b) En segundo lugar, el modelo del no intervencionismo tolerante que postula la tesis defendida por Kukathas (2003: 74-116; y 2012: 34-56), de que los grupos tienen derecho a preservar la cultura siempre que se garantice el derecho de salida;

c) Aquellas posiciones que pretenden compatibilizar algunos de los valores liberales con la protección de la diferencia cultural. En este grupo de respuestas encontraríamos

13 Shachar describe correctamente, mediante lo que denomina la paradoja de la vulnerabilidad multicultural el miedo feminista ante la potencial opresión que en nombre de los derechos de grupo pueden sufrir las mujeres cuando los esfuerzos bien intencionados dirigidos a mejorar la autonomía de los grupos y la diversidad cultural pueden hacer más gravoso revertir las desigualdades de género o, incluso, profundizar la subordinación. Véase Shachar (2001: 4-10). Cfr. Torbisco (2011). 
dos opciones que difieren entre sí en el modo en que debería articularse esa alternativa intermedia: (c.1.) Kymlicka, quien defiende la conocida distinción entre protecciones externas y restricciones internas (1996: 57-76, 152-164; 2003: 59, 75 y 76); y (c.2.) Parekh, quien plantea que -debido a que las culturas son imperfectas, limitadas, internamente plurales, y permeables a influencias externas-la promoción del diálogo entre ellas sería mutuamente beneficioso, tanto para los grupos como para los individuos (Cfr. Pérez de la Fuente, 2004: 399-430; y Torbisco, 2011).

Aquí solo me concentraré en el tercer grupo de respuestas argumentando que ni la posición de Kymlicka ni la de Parekh son satisfactorias. El problema de filósofo canadiense es que su teoría se centra en una concepción más bien esencialista del término "cultura"14, lo que impacta en la nítida distinción y en el diverso peso moral que tendrían las demandas de dos tipos de minorías: las nacionales y los inmigrantes (Parekh, 2006: 99-109). Estos últimos no estarían habilitados para ejercitar (y debiesen dejar de demandar) el derecho al autogobierno y a su autonomía cultural, aunque sí pueden exigir la mantención de algunas de sus prácticas asociadas a su identidad etnocultural que se traducirían en diversas facultades y exenciones que Kymlicka llama derechos de acomodo (2003: 75 y 76).

La cuestión clave es que no existe razón de peso para hacer tal distinción, al contrario, esa clasificación no hace más que reflejar una larga tendencia histórica liberal de delinear, por un lado, un claro contraste entre los grupos étnicos y las naciones, privilegiando a las segundas; $y$, por otro, establece una clara diferencia entre inmigrantes y ciudadanos (Parekh, 2006: 103). Apoyándose en lo mismo, otros han criticado la rigidez del planteamiento al ocuparse solamente de los casos antagónicos omitiendo las situaciones intermedias, como sería el caso de grupos oprimidos que, sin pertenecer a aquellas minorías en las que se concentra Kymlicka, sí requerirían que se les reconocieran algunos de los derechos de acomodo (Carens, 2000; y Young: 1997). Me parece que sería el caso, sin duda, de las minorías sexuales y las mujeres. ${ }^{15}$

El problema de la teoría de Parekh, por su parte, es su extrema ambigüedad. Esperar a que el debate fluya para que se haga más amigable no es algo que algunos grupos oprimidos -las mujeres, por cierto- estén dispuestos a hacer, pues su situación de explotación requiere respuestas normativas urgentes (Parekh, 2006: 295-335). Lo que debe dilucidarse, en mi opinión, es si la apelación a la cultura es una justificación válida

En las propias palabras de Kymlicka: “los Estados modernos desarrollan y consolidan invariablemente lo que yo llamo una 'cultura societal' -esto es, un conjunto de instituciones que abarcan tanto la vida pública como la vida privada, provistas de una lengua común que se ha desarrollado históricamente a lo largo del tiempo en un territorio dado y que proporciona a las personas una amplia gama de opciones respecto de cómo encauzar sus vidas. La aparición de una cultura societal -que requiere la normalización y difusión de una lengua común, así como la creación y la propagación de instituciones educativas, políticas y legales comunes- es un rasgo de modernización, pero es también algo que recibe un apoyo activo por parte del Estado" (2003: 78).

15 El otro aspecto débil de la tesis de Kymlicka es que no tiene una respuesta persuasiva para aquellos casos donde grupos no liberales pretendieran imponer restricciones internas a sus miembros, algo que el multiculturalismo liberal jamás aceptaría. Kymlicka arguye que sus críticos exageran, pues “cualquier solución duradera exigirá diálogo" (2003: 91). Lamentablemente, el filósofo canadiense no desarrolla en qué condiciones podría darse ese diálogo. 
para no aplicar un catálogo de derechos básicos y, en términos más abstractos, si existen razones persuasivas para no distribuir con pretensiones de universalidad el valor de la autonomía.

Si defendemos una posición multiculturalista nos encontraríamos en medio del dilema de considerar como válidas aquellas culturas -desgraciadamente demasiadas desde nuestra propia cosmovisión- que suponen la discriminación de la mujer o, en cambio, sostener normativamente la igualdad de la mujer. Pero en este último caso apelaríamos a valores de moralidad crítica universales que no todas las versiones del multiculturalismo podrían justificar ¿Se puede superar esta paradoja? Creo que sí. Si se concede que una cultura puede justificar la no aplicación de los derechos básicos a un grupo determinado -en el caso que nos ocupa, las mujeres- estaríamos dejándonos atrapar por lo que Benhabib denomina una sociología reduccionista de la cultura que se funda en premisas epistémicas falsas. ${ }^{16}$

En vez de eso, la filósofa nos propone una visión de la cultura (consistente con la presentada más arriba) articulada sobre la base del constructivismo social que escapa del esencialismo -que es más consistente con la propia visión multicultural-caracterizada por una visión narrativa de las acciones y de la cultura en donde debemos distinguir entre el punto de vista del observador social y el del agente social. El observador social es el que impone -de la mano de las élites locales- la unidad y la coherencia respecto de las culturas como entidades observadas. En cambio, el agente social representa la perspectiva de los participantes de la cultura que experimentan sus tradiciones mediante relatos narrativos compartidos, aunque también controvertidos y rebatibles. Desde su interior una cultura nunca aparece como un todo homogéneo (Benhabib, 2002: 4, 5). Pues bien, de esa concepción estática y tosca de la cultura las tesis multiculturalistas pretenden sacudirse: si una cultura cae en este vicio estaría siendo inconsistente con la única versión plausible del multiculturalismo, pues petrificaría la cultura a costa de la autonomía de algunos de sus miembros impidiendo que estos cuestionen su pertenencia a la cultura. La cultura dejaría de ser, como nos asegura Parekh, permeable e influenciable externamente haciendo que todo cuestionamiento sobre el modo cuando debemos enfrentar el desafío multicultural sea vano.

Pero no solo basta el reconocimiento de esa capacidad de revisión mediante el derecho de salida, ya que este traslada todo el peso del conflicto al individuo, excusando al grupo y al Estado. Si la identidad cultural de verdad interesa a los sujetos, será necesario considerar la posibilidad de que estos decidan permanecer en sus grupos desafiando a las élites del mismo o desechen la posibilidad de renunciar por el acentuado compromiso normativo con su grupo cultural o religioso (Phillips, 2007a: 151-157; y Shachar, 2001: 40-42). Luego, volviendo a Benhabib, la autora propone un modelo de democracia deliberativa como el más adecuado para responder al desafío multicultural. Este debe caracterizarse por dos

16 Las premisas epistémicas falsas son las siguientes: a) afirmar que las culturas son claramente delineables; b) creer que es posible realizar una descripción no controvertida de la cultura; y c) considerar que aun cuando las culturas y los grupos no se corresponden con exactitud entre sí, esto no implica problemas relevantes para la política. Benhabib (2002: 4). 
elementos claves: primero, un marcado énfasis en la esfera pública que es el lugar donde se sitúan las disputas multiculturales y donde también ocurren las transformaciones políticas y morales; y, segundo, una defensa de la apertura de la agenda del debate público difuminando la distinción entre lo público y lo privado, entre lo que es de todos y lo que es solo propio de mi cultura (Benhabib, 2002: 106-114; y Deveaux, 2000: 138-179).

En fin, si el diálogo intercultural y entre grupos surge al interior de una adecuada concepción de la democracia deliberativa que defienda una visión no reduccionista de la cultura, podremos transitar del monismo excluyente de la diversidad -que considera a otras formas de vida inferiores fruto de la ignorancia y de la barbarie- al pluralismo de valores que sostiene que existen ciertos valores universales pero diversas formas de vida que los pueden encarnar culturalmente.

\section{APROXIMACIÓN CRÍTICA A LA TESIS DE LAS JURISDICCIONES MULTICULTURALES Y LA DEMOCRACIA DELIBERATIVA COMO TRASFONDO}

Según las cifras del Servicio Nacional de la Mujer, en la Región de La Araucanía son 17 los casos en los que se han resuelto demandas de violencia de género, entre noviembre de 2011 y 2012, con una disculpa del agresor con arreglo a los artículos 9 y 10 del Convenio 169 de la OIT y según dicta la costumbre mapuche. Se trata de familias de etnia mapuche en los que la defensoría penal invoca las formas propias que tiene ese pueblo para resolver los conflictos de violencia intrafamiliar por medio de un "acuerdo reparatorio" que supone disculpas públicas ante las autoridades correspondientes de la etnia. ¿Pero cómo se logra administrar la superposición intercultural de jurisdicciones en este caso? Bajo la regla de que los acuerdos reparatorios solo se aceptan si se trata de mujeres que no han sufrido violencia histórica o reiterada, ni lesiones graves. En estos últimos casos, la defensoría comprendería que tal nivel de violencia no permite el progreso de dicha salida alternativa. ${ }^{17}$

El caso expuesto puede conectarse con la propuesta de las jurisdicciones multiculturales de Shachar ${ }^{18}$ que me interesa explorar. Este modelo opera del siguiente modo: protege los grupos vulnerables $\mathrm{u}$ oprimidos por medio del entrecruzamiento de las jurisdicciones estatales y locales (o grupales) desde una concepción dinámica de las culturas. Así se justificarían las acomodaciones de la jurisdicción estatal que mejoran la posición de los grupos subordinados, mientras se amparan las diferencias culturales, generando condiciones para el cambio interno de la cultura y no fomentando su oposición refractaria hacia la comunidad dominante. Se institucionaliza una regla de no monopolio jurisdiccional y se

17 “Tribunales validan disculpas verbales ante violencia intrafamiliar entre Mapuches", Millecer Bravo. En: http:/ / www.lexweb.cl/tribunales-validan-disculpas-verbales-ante-violencia-intrafamiliar-entre-mapuches

18 Una propuesta que puede enmarcarse, en general, dentro de aquellas que defienden un modelo constitucional e institucional plurinacional, pero con la ventaja de que la tesis de Shachar puede aplicarse no solo a minorías nacionales, sino a toda clase de minorías que demanden algún reconocimiento de sus diferencias culturales. Cfr. Tierney (2004). 
asegura la elección individual, caso a caso, de la ley aplicable. De modo que, por ejemplo, una mujer indígena tendrá asegurado jurisdiccionalmente sus derechos individuales cuando escoja someterse a la jurisdicción estatal en vez de la costumbre indígena que la discrimine. La idea es equilibrar, por un lado, el reconocimiento de la autonomía normativa del grupo cultural y, por otro, la operatividad de los mecanismos previstos para la protección de los derechos fundamentales (Shachar, 2001: 117ss.; y Pérez de la Fuente, 2004: 423-425).

Más allá de la crítica obvia a este modelo por su falta de certeza y por el costo que supone implementar una jurisdicción pluralista, quisiera evaluar moralmente el modelo de Sachar (Cfr. Sánchez, 2012: 84-86). En primer lugar, uno de los problemas del pluralismo es que no se traza una distinción clara entre lo normativamente correcto y lo institucionalmente factible, es decir, podemos llegar a confundir la prudencia moral, la perspicacia política, el ingenio y la comprensión estratégica de cuestiones morales y políticas con aquello que por principio consideramos justo. La justicia nos demanda imparcialidad para determinar aquello que es mejor para todos los que consideramos seres morales igualmente valiosos, con independencia de su pertenencia cultural.

En segundo lugar, los procedimientos necesarios para institucionalizar la jurisdicción multicultural a veces corren el serio riesgo de provocar una especie de refeudalización de la legislación, socavando la igualdad ante la ley (Benhabib, 2002: 128). Por supuesto, la crítica multiculturalista dirá que dicha igualdad es injusta, pero no debemos desconocer, de la mano de Barry (2002: 38-50), que hay una diferencia relevante entre las excepciones hechas a favor de determinados grupos, prácticas e instituciones sobre la base de razones que se supone todos comparten, y el presupuesto de que las razones que justificarían el trato diferencial ya no exijan ser generalizadas. O sea, si no se especifica la capacidad de los principios constitucionales para prevalecer por sobre otro tipo de normativas jurídicas, es posible que no estemos resolviendo la paradoja de la vulnerabilidad multicultural, sino simplemente permitiendo su recirculación infinita en todo el sistema jurídico.

En tercer lugar, otro peligro de la propuesta de Shachar es caer en una especie de endiosamiento del proceso jurídico judicializando el diálogo político y cultural. En vez de eso, es indispensable que el multiculturalismo, precisamente porque en muchas de sus manifestaciones desafía los presupuestos básicos de las democracias liberales, libere su potencial conflictivo en la esfera civil pública mediante el diálogo, la controversia y la negociación entre ciudadanos y ciudadanas comunes (Benhabib, 2002: 115).

En una democracia multicultural vibrante, las hábiles maniobras de los abogados ejercitando las herramientas del modelo de las jurisdicciones multiculturales no deberían reprimir del todo el conflicto cultural y político, ni el aprendizaje por medio del conflicto. Al contrario, tienen que ser los propios ciudadanos democráticos -y no solo los jueces y legisladores- los que deben aprender a convivir bajo los siguientes principios básicos de una democracia deliberativa:

Primero, la reciprocidad igualitaria y principio de la inclusión política, que demandan que la pertenencia de un individuo a una determinada minoría no se traduzca en el otorgamiento de un grado inferior de derechos civiles, políticos, económicos o culturales en relación con los que disfruta la mayoría. 
Segundo, la autoadscripción al grupo: una persona no debe ser asignada automáticamente a un grupo cultural, religioso o lingüístico en virtud de su nacimiento. La pertenencia grupal de una persona debe admitir las formas más amplias de autoadscripción y autoidentificación posibles. Existirán muchos casos en que tales autoidentificaciones serán impugnadas, pero el Estado no debe simplemente garantizar al grupo el derecho para definir y controlar la membresía a expensas del individuo. Es deseable además que en algún punto de la vida adulta de los individuos se les consulte acerca de su deseo de continuar siendo parte de sus comunidades de origen.

Tercero, la libertad de salida y asociación de un determinado grupo cultural debe ser irrestricta, aunque ello pueda conllevar la pérdida de ciertos privilegios, los que en todo caso deben ser posibles de ser judicializados para su potencial control. Además se debe velar por el principio de la no dominación, cuya finalidad es evitar que los participantes, especialmente aquellos con mayor poder económico o social (los hombres), coaccionen a otros (las mujeres) socavando la deliberación política y excluyendo a ciertos individuos del proceso.

Finalmente, el principio de la posibilidad de revisión aboga porque una vez alcanzado un compromiso o un acuerdo debe ser posible revisarlo cuando sea necesario. Este principio facilita que las personas y los grupos estén abiertos a suscribir un pacto, pues conlleva implícitamente la posibilidad de renegociar, haciéndose cargo de esta forma del carácter fluido de las prácticas sociales y culturales (Cfr. Benhabib, 2002: 131-132).

\section{CONCLUSIONES}

Tomando en consideración las anteriores prevenciones, el modelo de las jurisdicciones multiculturales podría constituir un mecanismo institucional y jurídico satisfactorio para arbitrar las tensiones y disputas entre una teoría de los derechos que pretende distribuir universal e igualitariamente ciertos derechos básicos y el debido respeto a las dinámicas identitarias internas de cada cultura, siempre y cuando se inserte en una adecuada concepción de la democracia deliberativa.

Conectemos el modelo de Shachar con el caso de la violencia de género contra mujeres mapuche. ${ }^{19}$ Es evidente que la política seguida por la defensoría caso a caso constituye nada más un esbozo muy rudimentario de un modelo de jurisdicciones multiculturales. Para que realmente operara debieran cumplirse copulativamente las siguientes condiciones:

a) Institucionalizar jurídicamente el carácter nomoi no solo de la comunidad dominante, sino también de la comunidad minoritaria mapuche, por un reconocimiento general de potestades normativas que dé lugar a una genuina jurisdicción pluralista que excede, por cierto, la muy limitada aplicación de los artículos 9 y 10 del Convenio 169 de la OIT;

19 Cfr. Bolados (2010). Desde una matriz teórica diferente vale la pena revisar este muy exhaustivo estudio de las relaciones entre multiculturalismo, neoliberalismo y etnicidad asociados a la política indígena e intercultural en salud implementada por el Estado chileno en los últimos años. 
b) Establecer mecanismos institucionales que aseguren la igual participación política de la nación mapuche dentro de la comunidad política mayor entendida como Estado plurinacional, que permitan autonomía territorial y funcional; única forma de administrar establemente el hecho indesmentible de que la identidad mapuche se construye en la intersección de la identidad general y comunitaria-local; y

c) Asegurar la posibilidad de elección de la mujer mapuche de someterse a la jurisdicción local o la chilena caso a caso en un contexto en el que se cumplen todas y cada una de las condiciones básicas de una democracia deliberativa expuestas en el apartado anterior.

Cumplidas las condiciones anteriores, es esperable que los individuos que pertenecen a la comunidad dominante y la minoritaria se autoconciban como agentes morales cuya identidad tiene una afiliación plural permitiendo que, en la vida cotidiana, tanto el Estado como los grupos minoritarios se comprendan como entes sociales mutables que se influyen recíprocamente velando por sus miembros (Cfr. Shachar, 2001: 118).

Según todo lo anterior, parece posible aplicar herramientas como el modelo de las jurisdicciones multiculturales que faciliten el entrecruzamiento de las tesis normativas liberales más fundamentales (paradigmáticamente la distribución igualitaria de los derechos individuales más básicos) y los presupuestos metaéticos de cada cultura. Esa empresa la considero tan deseable como viable. ¿Acaso las demandas democráticas en Medio Oriente o Noráfrica no han nacido en el seno de las culturas locales? Eso demostraría tres cosas: una, los valores democráticos tienen un potencial transcultural; dos, no sería acertada la creencia esencialista de que las culturas no occidentales son incapaces de hacer suyos esos valores democráticos por ser totalmente incompatibles con sus horizontes de sentido común; y, tres, las culturas son permeables, porosas y admiten la disidencia interna, siendo la distribución del valor de la autonomía la única forma de asegurar esa disidencia vernácula.

En fin, si todo lo dicho es plausible, podrían sentarse, incluso, las bases para la construcción de un cosmopolitismo interactivo que permita renegociar los derechos individuales de pertenencia a un demos determinado, por medio de la aplicación de los principios de una ética discursiva y prácticas democráticas contextualizadas, que incluyan en una conversación moral trasnacional a los pueblos indígenas o a los inmigrantes (Benhabib, 2005 y 2006a; Sánchez, 2007: 63-70; y Sánchez, 2008: 269-307). En fin, la circunstancia de que los grupos culturales puedan sobrevivir como entidades diferenciadas bajo estas condiciones es una pregunta abierta y contingente, pero creo que son necesarias si el modelo de las jurisdicciones multiculturales desea conciliar la protección de la diversidad cultural y la igualdad democrática, sin por ello comprometer los derechos de los que siempre son sus más débiles miembros: las mujeres y los niños que, voluntaria o involuntariamente, casi siempre las acompañan.

\section{REFERENCIAS}

Amorós, Celia. 1997. Tiempo de feminismo. Sobre feminismo, proyecto ilustrado y postmodernidad. Madrid: Cátedra. Banting, Keith y Kymlicka, Will. ed. 2002. Multiculturalism and the Welfare State: Recognition and Redistribution in Contemporary Democracies. Oxford: Oxford University Press. 
Barry, Brian. 2002. Culture and Equality. Harvard: Harvard University Press.

Benhabib, Seyla. 2002. The Claims of Culture. Equality and Diversity in the Global Era. Princeton: Princeton University Press.

Benhabib, Seyla. 2005. Los derechos de los otros. Extranjeros, residentes y ciudadanos (traducción de Gabriel Zadunaisky). Barcelona: Gedisa.

Benhabib, Seyla. 2006a. Another Cosmopolitanism. Oxford: Oxford University Press.

Benhabib, Seyla. 2006b. El ser y el otro en la ética contemporánea. Feminismo, comunitarismo y posmodermismo (traducción de Gabriel Zadunaisky). Barcelona: Gedisa.

Bolados, Paola. 2010. “Neoliberalismo multicultural en el Chile democrático: Gubernamentalizando la salud atacameña a través de la participación y el etnodesarrollo". Tesis para optar al grado de Doctora en Antropología, Universidad Católica del Norte - Universidad de Tarapacá. Disponible en: http:/ / dspace2.conicyt.cl/handle/10533/89117?show=full

Carens, Joseph H. 2000. Culture, Citizenship, and Community. A Contextual Exploration of Justice as Evenhandedness. Oxford: Oxford University Press.

Delanty, Gerard. 2010. Community (2 ed.). Londres: Routledge.

Deveaux, Monique. 2000. Cultural Pluralism and Dilemmas of Justice. New York: Cornell University Press.

Deveaux, Monique. 2006. Gender and Justice in Multicultural Liberal States. Oxford: Oxford University Press.

De Lucas, Javier. 2001. "La(s) sociedad(es) multicultural(es) y los conflictos políticos y jurídicos". En La multiculturalidad. Madrid: Consejo General del Poder Judicial, 102-61.

Dhamoon, Rita. 2007. "The Politics of Cultural Contestation". En: Sexual, Justice/Cultural Justice. Critical Perspectives in Political Theories and Practice, editado por Barbara Arneil, Monique Deveaux, Rita Dhamoon y Avigail Eisenberg. Oxford: Routledge.

Fraser, Nancy. 2010. "Rethinking Recognition". New Left Review 3: 120-107. Disponible en: http://www. newleftreview.org $/$ ? view $=2248$.

Frazer, Elizabeth y Lacey, Nicola. 1993. The Politics of Community. A Feminist Critique of the LiberalCommunitarian Debate. Toronto: University of Toronto Press.

Guerra, María J. 2000. “Una apuesta por el feminismo global”. En Leviatán: Revista de Hechos e Ideas 80: 116-101.

Habermas, Jürgen. 1999. "La lucha por el reconocimiento en el Estado democrático de derecho". En La inclusión del otro, 227-189 (traducción de Juan Carlos Velasco). Barcelona: Paidós

Habermas, Jürgen. 2003. "De la tolerancia religiosa a los derechos culturales". En Claves de razón práctica 129: 10-4.

Hesse- Biber, Sharlene (ed.). 2012. The Handbook of Feminist Research. Theory and Praxis (2 ed.). Los Ángeles (California): SAGE Publications.

Jackson Preece, Jennifer. 2005. Minority Rights. Cambridge: Polity Press.

Kymlicka, Will. 1996. Ciudadanía multicultural. Una teoría liberal de los derechos de las minorías (traducción de Auleda Castells). Barcelona: Paidós.

Kymlicka, Will. 2002. Contemporary Political Philosophy. An Introduction (2 ed.). Oxford: Oxford University Press.

Kymlicka, Will. 2003. La política vernácula. Nacionalismo, multiculturalismo y ciudadanía (traducción de Tomás Fernández Aúz y Beatriz Eguilbar). Barcelona: Paidós.

Kymlicka, Will. 2007. Multicultural Odysseys. Navigating the New International Politics of Diversity. Oxford: Oxford University Press.

Kukathas, Chandra. 2003. The Liberal Archipelago. A Theory of Diversity and Freedom. Oxford: Oxford University Press.

Kukathas, Chandra. 2012. "Exit, Freedom and Gender". En On Exit: Interdisciplinary Perspectives on the Right of Exit in Liberal Multicultural Societies, editado por Dagmar Borchers y Annamari Vitikainen, 56-34. Berlin: Walter de Gruyter.

Modood, Tariq. 2007. Multiculturalism. A Civic Idea. Polity Press.

Nussbaum, Martha. C. 1999. "A Plea for Difficulty", en Is Multiculturalism Bad for Women? Susan Moller Okin with Respondents, editado por Joshua Cohen, Matthew Howard, y Martha C. Nussbaum, , 114105. Princeton: Princeton University Press.

Okin, Susan M. 1998. “Feminism and Multiculturalism: Some Tensions". Ethics 108 (4): 684-661. 
Okin, Susan M. 1999. Is Multiculturalism Bad for Women? Susan Moller Okin with Respondents, editado por Joshua Cohen, Matthew Howard, y Martha C. Nussbaum. Princeton: Princeton University Press. [originalmente publicado con el mismo título en 1997. Boston Review, 22, Octubre/Noviembre. Disponible en: http://bostonreview.net/BR22.5/okin.html].

Okin, Susan M. 2005. "Multiculturalism and feminism: no simple question, no simple answers". En Minorities within Minorities. Equality, Rights and Diversity, editado por Avigail Einsenberg y Jeff Spinner-Halev, 89-67. Cambridge: Cambridge University Press.

Parekh, Bhikhu. 1999. "Political Theory and the Multicultural Society". Radical Philosophy 95. Disponible en: http:/ / www.radicalphilosophy.com/default.asp?channel_id=2188\&editorial_id=10166.

Parekh, Bhikhu. 2002. "Barry and the dangers of liberalism". En Multiculturalism Reconsidered. Culture and Equality and its Critics, editado por Paul Kelly, 150-133. Cambridge: Polity Press.

Parekh, Bhikhu. 2005. "A Varied Moral World". En Is Multiculturalism Bad for Women? Susan Moller Okin with Respondents, editado por Joshua Cohen, Matthew Howard, y Martha C. Nussbaum, 75-69. Princeton: Princeton University Press.

Parekh, Bhikhu. 2006. Rethinking Multiculturalism. Cultural Diversity and Political Theory (2 ed.). Basingstoke: Palgrave Macmillan.

Pérez de la Fuente, Oscar. 2004. "Indígenas y derechos colectivos: ¿es el multiculturalismo malo para las mujeres?". Derechos y libertades, Revista del Instituto Bartolomé de las Casas 13: 430-399.

Pérez de la Fuente, Oscar. 2005. Pluralismo cultural y derechos de las minorías. Madrid: Dykinson.

Phillips, Anne. 2007a. Multiculturalism without Culture. Princeton: Princeton University Press.

Phillips, Anne. 2007b. "What is 'Culture'?". En Sexual, Justice/Cultural Justice. Critical Perspectives in Political Theories and Practice, editado por Barbara Arneil, Monique Deveaux, Rita Dhamoon y Avigail Eisenberg, 15-29. Oxford: Routledge.

Rawls, John. 1971. A Theory of Justice. Oxford: Oxford University Press.

Rawls, John. 1996. El liberalismo político (traducción de Antoni Domènech). Barcelona: Crítica.

Rawls, John. 1999. Justicia como equidad. Materiales para una teoría de la justicia (traducción de Miguel Ángel Rodilla). Madrid: Tecnos.

Rawls, John. 2002. La Justicia como equidad. Una reformulación (traducción de Andrés de Francisco). Barcelona: Paidós.

Sánchez, Cristina. 2002. "Feminismo y ciudadanía". En Estado, justicia, derechos, editado por Elías Díaz y José Luis Colomer, 370-347. Madrid: Alianza.

Sánchez, Cristina. 2005. "Género y constitución: un análisis de la ciudadanía social de las mujeres". En Constitución y derechos fundamentales: 25 aniversario de la constitución, editado por Francisco Laporta et al., 605-586. Madrid: Centro de Estudios Constitucionales y Políticos.

Sánchez, Cristina. 2007. “Renegociando la ciudadanía: las prácticas de iteraciones democráticas”. En Multiculturalismo y feminismo, 70-63. Madrid: Instituto de la Mujer (Ministerio de Trabajo y Asuntos Sociales).

Sánchez, Cristina. 2008. "Seyla Benhabib: hacia un universalismo interactivo". En Teorías políticas contemporáneas, 307-269. Valencia: Tirant lo Blanch.

Sánchez, Cristina. 2012. "Género y cultura(s): aportaciones a un debate desde la democracia deliberativa". En Género, cultura y sociedad, 93-71. México D. F.: SCJN-Fontamara.

Selamé, Nicole y Villavicencio Miranda, Luis. 2011. "Liberalismo, multiculturalismo y Estado de bienestar". Ideas y Valores. Revista Colombiana de Filosofía, Universidad Nacional de Colombia 60 (147): 140-111. Disponible en: http:/ / www.revistas.unal.edu.co/index.php/idval/article/viewFile/36752/38704.

Shachar, Ayelet. 2001. Multicultural Jurisdictions. Cultural Differences and Women's Rights. Cambridge: Cambridge University Press.

Song, Sarah. 2007. Justice, Gender, and the Politics of Multiculturalism. Cambridge: Cambridge University Press. Squires, Judith. 2006. "Culture, Equality and Diversity". En Multiculturalism Reconsidered. Culture and Equality and its Critics, editado por Paul Kelly, 132-114. Cambridge: Polity Press.

Tierney, Stephen. 2004. Constitutional Law and National Pluralism. Oxford: Oxford University Press.

Torbisco, Neus. 2006. Group Rights as Human Rights. A Liberal Approach to Multiculturalism. Dordrecht: Springer.

Torbisco, Neus. 2011. "Beyond the Feminism vs. Multiculturalism Dispute on Group Rights: Towards a Participatory Approach". Documento para la discusión, SELA (inédito). Disponible en: http://www. law.yale.edu/documents/pdf/sela/SELA11_Torbisco_CV_Eng_20110524.pdf. 
Villavicencio Miranda, Luis. 2007. “La concepción política de la persona y las visiones constitutivas del bien". Ideas y Valores. Revista Colombiana de Filosofía, Universidad Nacional de Colombia 135: 49-29. Disponible en: http://www.revistas.unal.edu.co/index.php/idval/article/view/8620.

Villavicencio Miranda, Luis. 2009. "Algunas críticas a la idea de razón pública rawlsiana". Revista de Derecho de la Pontificia Universidad Católica de Valparaíso 32 (1): 557-533. Disponible en: http:/ / www. scielo.cl/scielo.php?script=sci_arttext\&pid=S0718-68512009000100015\&lng=es\&nrm=iso\&tlng=es.

Villavicencio Miranda, Luis. 2010a. "La relevancia moral de la identidad cultural". Polis 9 (26). Disponible en: http:/ /www.scielo.cl/scielo.php?script=sci_arttext\&pid=S0718-65682010000200019\&lng=e s\&nrm=iso\&tlng=es.

Villavicencio Miranda, Luis. 2010b. "Privatizando la diferencia: El liberalismo igualitario y el pluralismo cultural". Revista de Derecho (Valdivia) XXIII (1): 57-37. Disponible en: http:/ /www.scielo.cl/scielo. php?script=sci_arttext\&pid=S0718-09502010000100002\&lng=es\&nrm=iso\&tlng=es.

Villavicencio Miranda, Luis. 2012. “Son reconciliables las demandas de género con el multiculturalismo? La apuesta por un cosmopolitismo sensible a la diferencia". En Actas del Congreso Internacional de Derecho. En homenaje al centenario de la escuela de derecho, Tomo I. Valparaíso: EDEVAL, 545-519.

Walzer, Michael. 1993. Las esferas de la justicia. Una defensa del pluralismo y la igualdad (traducción de Heriberto Rubio). México D. F.: Fondo de Cultura Económica.

Young, Iris M. 1997. "A Multicultural Continuum: A Critique of Will Kymlicka's Ethnic-Nation Dichotomy". Constellations 4 (1): 53-48.

Young, Iris M. 2000. La justicia y la política de la diferencia (traducción de Silvina Álvarez). Madrid: Cátedra. Zúñiga, Yanira. 2005. "Democracia paritaria: de la teoría a la práctica". Revista de Derecho (Valdivia) XVIII (2): 154-131. Disponible en: http://www.scielo.cl/scielo.php?script=sci_arttext\&pid=S071809502005000200006\&lang=pt.

Zúñiga, Yanira. 2006. “El derecho como representación-deformación. Un análisis de su construcción adversarial en clave de género y derechos fundamentales". Revista de Derecho (Valdivia) XIX (1): 59-35. Disponible en: http:/ / www.scielo.cl/scielo.php?script=sci_arttext\&pid=S0718-09502006000100002\&lang=pt.

Zúñiga, Yanira. 2009. “La 'generización' de la ciudadanía. Apuntes sobre el rol de la diferencia sexual en el pensamiento feminista". Revista de Derecho (Valdivia) XXII (2): 64-39. Disponible en: http:/ / www. scielo.cl/scielo.php?script=sci_arttext\&pid=S0718-09502009000200003\&lang=pt.

Zúñiga, Yanira. 2010. “Ciudadanía y género. Representaciones y conceptualizaciones en el pensamiento moderno y contemporáneo". Revista de Derecho Universidad Católica del Norte 17(2): 163-133. Disponible en: http:/ / www.scielo.cl/scielo.php?script=sci_arttext\&pid=S0718-97532010000200006\&lang=pt.

Luis Villavicencio es Doctor en Derecho por la Universidad Autónoma de Madrid. Abogado. Licenciado en Ciencias Jurídicas y Sociales de la Universidad Diego Portales. Académico e investigador de la Universidad de Valparaíso. Es miembro del directorio de la Sociedad Chilena de Filosofía Jurídica y Social. Es coautor (junto con Agustín Squella y Alejandra Zúñiga) del libro Curso de filosofía del derecho. Santiago: Editorial Jurídica de Chile. 2012. E-mail: luis.villavicencio@uv.cl 
